\title{
Correction to: Patient attitudes toward pooled surgical waitlists in urogynecology
}

\section{Rebekah A. Zee ${ }^{1} \cdot$ Aisling A. Clancy ${ }^{1} \cdot$ Hisham Khalil ${ }^{1}$}

Published online: 7 December 2019

(C) The International Urogynecological Association 2019

\section{Correction to: International Urogynecology Journal} https://doi.org/10.1007/s00192-019-04050-4

Table 3 in the originally published article contains layout error. Corrected Table 3 shown below.

The online version of the original article can be found at https://doi.org/ 10.1007/s00192-019-04050-4

\section{Hisham Khalil}

hkhalil@toh.ca

1 Division of Urogynecology, Department of Obstetrics and

Gynecology, The Ottawa Hospital, 1967 Riverside Dr,

Ottawa, ON K1H 1A2, Canada 
Table 3 Patient characteristics based on acceptance of a pooled surgical waitlist

Not accepting of a pooled surgical waitlist $(n=113)$
(4).2\%)

$41(36.3 \%)$

$30(26.5 \%)$

$44(38.9 \%)$

$29(25.7 \%)$

$4(3.5 \%)$

$2(1.8 \%)$

$3(2.7 \%)$

$1(0.9 \%)$

$2(1.8 \%)$

$52(46.0 \%)$

$39(34.5 \%)$

$19(16.8 \%)$

$28(24.8 \%)$

$69(61.1 \%)$

$13(11.5 \%)$

$1(0.9 \%)$

$1(0.9 \%)$

$1(0.9 \%)$
Accepting of a pooled surgical wait list $(n=60)$

$7(11.9 \%)$

$20(33.9 \%)$

$32(54.2 \%)$

$P$

0.071

$14(23.3 \%)$

$25(41.7 \%)$

$15(25.0 \%)$

$3(5.0 \%)$

$2(3.3 \%)$

$0(0.0 \%)$

$0(0.0 \%)$

$1(1.7 \%)$

$26(43.3 \%)$

$25(41.7 \%)$

$8(13.3 \%)$

$29(49.2 \%)$

$25(42.4 \%)$

$4(6.8 \%)$

$0(0.0 \%)$

$0(0.0 \%)$

$1(1.7 \%)$

MUS mid-urethral sling; vag hyst vaginal hysterectomy

Publisher's note Springer Nature remains neutral with regard to jurisdictional claims in published maps and institutional affiliations. 